\title{
Quality Framework Performance in One Year of COVID-19 Restrictions in a Higher Education Institute
}

\author{
Paul O'Leary \\ Waterford Institute of Technology \\ Derek O'Byrne \\ Waterford Institute of Technology
}

\begin{abstract}
The entire academic year 2020-21 was completed under COVID-19 restrictions. This created challenges for students and staff, and to the quality frameworks that underpin the higher education (HE) being offered. Higher education (HE) quality frameworks were tested in a manner that had not been foreseen on their creation.

This work examines the performance of one HE framework, which was created long before national lockdowns or social distancing, after an entire academic year of 2020-21.

Critical evaluation will be offered by the authors, by external examiners, from student surveys and from an institutional quality review panel which took place in the academic year. The aim is to evaluate if the quality framework was sufficiently robust to offer our students a quality education experience, to evaluate the impact of some operational modifications and to reassure the public in terms of the standard of our graduates.
\end{abstract}

Keywords: higher education, quality framework, pandemic performance

\section{INTRODUCTION}

Since early 2020 COVID-19 has had an immense impact on higher education. Research on COVID-19 carried out since then has examined the impact on higher education in general (Marinoni, van't Land, \& Jense, 2020), (Burki, June 2020), (Crawford, et al., 2020), on international mobility (Martel, 2020), on teaching \& learning (García-Peñalvo, Corell, Abella-García, \& Grande, 2020), (Kumar Arora \& Srinivasan, 2020), (Kumar, 2020), (Mishra, Gupta, \& Shree, 2020), on research (Lancet, 2020) and the positive outcomes for HE that may emanate post-COVID (Toquero, 2020), (Neuwirth, Jovic, \& Mukherji, 2020). However, literature on the quality aspects of higher education has focused on the educational experience for staff and students (Ramírez-Hurtado, Hernández-Díaz, López-Sánchez, \& Pérez-León, 2021), education services (Camilleri, 2021) or the quality of life of the principle actors (Du, et al., 2021). The overall quality framework performance has not been published, as far as the authors can ascertain, except for a paper by the authors evaluating the performance of the quality framework in their own HEI in the early response to the pandemic, at the end of the 2019-20 academic year (O'Leary \& O'Byrne, June, 2021). In this paper the 
authors examine the quality framework's performance in our own institute over the entire 2020-21 academic year (AY '20-'21). Critical evaluation will be offered by the authors, by external examiners, from student surveys and from an institutional quality review panel which took place in AY '20-'21.

Waterford Institute of Technology is a higher education institute, located in the South-East of Ireland, of circa 7,500 full and 1,500 part-time students with programs ranging from short cycle undergraduate to doctoral degrees. The Institute, as a result of COVID-19, was forced to pivot rapidly to off-campus delivery and assessment for all of its activities from March 2020 and was forced to operate since then under the provisions of 'remote emergency teaching'. Remote emergency teaching provided that the maximum amount of teaching and assessment should be conducted through virtual activity rather than traditional oncampus modes. In effect this meant that all activity in the Institute was off-campus throughout AY '20-'21.

While the physical structures of the institute were closed, students, staff and the public had an expectation that the higher education would continue and would maintain the same high standards. This provided a significant challenge in ensuring the implementation and interpretation of the quality assurance and enhancement activities reflected the emerging realities of conducting so much remote activity.

The institute, in line with Irish society, hoped that some return to normality would emerge as the year unfolded and early signs that the second academic semester might see some return to on-campus activity were dashed, as an attempt to open society in late 2020 resulted in a further COVID wave and even more stringent lockdowns, resulting in Ireland having some of the tightest and longest lock-downs internationally. To respond to the challenges, new decision pathways and new organizational arrangements became important tools to ensure continuity of operations. This happened, not only in academic engagement but also in the institute's management processes, as well the institute's connection with external bodies and agencies. Of particular note was the institute's completion of the national quality assurance review process during the course of the year, through a virtual process.

By the end of the academic year the institute had successfully completed a full cycle of teaching, learning and assessment for all of its students. Programs had been delivered, assessed and grades award. Individual module results reflected traditional patterns of award distribution and ranges of awards consistent with previous years.

\section{QUALITY FRAMEWORK}

The institute's quality framework underpins every aspect of its performance, and it was therefore crucial that it operate well to successfully negotiate the 2020-21 academic year. While the quality framework pre-COVID operated in an ever-increasing, complex environment that provided for effective policies and procedures within predictable parameters, it could not ever have been developed anticipating the nature and extent of the complexity and change that subsequently ensured. The challenge of ensuring quality assurance and protecting the health and wellbeing of students and staff became a very real issue.

We propose to structure our discussion around six key framework elements of quality performance:

- Academic and Student-centered Values

- Staff and Student Quality Culture

- Informed Practice and External Engagement

- Proportional Implementation of Quality Processes

- Comprehensive, Transparent and Publicly Accountable QA

- Measurement

\section{Academic and Student-Centered Values}

For the most part in AY '20-'21 students were off-campus, with anticipated return dates being cancelled due to increases in COVID case numbers. The institute decided to prioritize the students' perspective from the beginning of the pandemic, with the students' union representing the student voice in discussions and surveys providing feedback from the student body. The students' union and institute co-developed a COVID-19 Student Charter, which described the responsibilities and commitments of the student body, the 
institute and students' union and explained what education would look like in the COVID-19 era. The charter particularly highlighted the need for strong communication in every respect, which was evaluated two months and again 14 months after the start of the first lockdown, through a survey of the entire student population. Adapting learning to a COVID-19 era was difficult, but it helped considerably to do so in a manner that was collaborative and engaging. However, survey results presented later in this paper indicate that the communications to the wider student body was initially not good enough and multi-faceted improvements were initiated as AY '20-'21 progressed. The external review panel commended the institute for the enhanced engagement and consultation between the executive and the students' union.

Another key part of embedding academic and student-centered values was created through an engaged academic council, the main deliberative body for academic matters, which increased their frequency of meetings, created dedicated sub-committees for particular work packages, and communicated widely throughout the institute, thus providing both a forum for debating issues and a legitimate rationale for actions connected to the quality framework.

The dynamic and complex nature of the COVID-19 challenge meant that some early decisions were made to support the staff and students that would only be temporary in nature. For example, the Academic Council decided that no student would be disadvantaged, as a result of the pandemic and therefore established a mitigation process, which included the possibility of alternative assessments and modified progression rules. While this solution successfully addressed the immediate problem at the pandemic outset, the modified progression rules were removed at the end of AY '20-'21, as some students were carrying too many incomplete modules. The external review panel recognized that the transition to emergency remote teaching was handled well but recommended more comprehensive approaches to the collection and analysis of information from alumni, students and staff about the delivery of programs and the student experience, to promote strategies for the continuing improvement of the quality of teaching.

Finally in terms of academic-centric values, the institute has a core objective of recruiting and developing high-quality academic staff and researchers. Therefore, training and guidelines were prepared to address the pressing needs of academic staff who had a dramatic change in their academic delivery and assessment. The dedicated Technology-Enhanced Learning unit significantly increased the number and nature of training workshops to support the academic staff development and the effective utilization of the eLearning infrastructure.

\section{Staff and Student Quality Culture}

The institute's quality culture under COVID-19 was successful due to three interrelated activities engaging staff, students and external stakeholders:

1. the sustained operation of cycles of development, implementation and review of policies and procedures that inform activity and initiated new policy, procedures and practices;

2. a defined structure that clearly articulated responsibility of key institute community members with respect to the quality assurance activities; and

3. continued publishing policies and procedures across the range of institute activities that were open for change by all stakeholders and eventually approved by the appropriate institutional body.

Initially the organization and communication of these activities was a challenge, especially as the institute community dealt with the infrastructure and broadband requirements of moving online. This quality culture was examined in a scheduled institutional review by an external panel of experts, with all participants communicating virtually. The original review was of course intended to be with the panel physically present on-campus, so there were concerns in relation to the operation of and capacity of a virtual review. However, notwithstanding the challenges and delays posed by COVID, the review meetings and communications before and after the external panel's virtual visit were well managed. The external panel examined the pre-COVID operation, the performance during the pandemic and the plans for the future. The panel's findings were broadly positive, but with recommendations to sustain ongoing improvements.

The scale and nature of the academic delivery and assessment changes were at levels heretofore never experienced. However, an example of the flexibility and resilience of the quality framework can be seen in 
the response to the challenge posed by students across the entire program portfolio struggling to achieve all credits required for stage advancement, sometimes for reasons related to the pandemic, remote learning or regional lockdowns. Academic Council decided to delegate this key decision to each program board. Therefore, program boards made decisions with students, in relation to students progressing, while carrying a larger than usually permitted number of credits. Program boards were empowered to permit students to progress carrying more than the previous maximum of 10 credits, subject to the Board being confident in the wisdom of each decision and to the Board informing the student of the challenge posed by carrying those modules.

\section{Informed Practice and External Engagement}

Engagement with all internal and external stakeholders is core to informing academic practice in learning, teaching, assessment and research; in collaboration; in economic and regional development; in establishing and realizing civic and community objectives and in introducing and sustaining internationalization.

Subject area external examiners are a key component in our assessment QA. In their reports they noted distinct mark variability in some modules compared to others. While they reported that the assessments were on the whole appropriate and fair, with a good variety of assessment methods, they looked for a longterm approach across the institute.

When the first national lockdown occurred, the institute was already on a merger path with another regional institute, with a plan to then apply for a technological university status. This initiative is driven in part by many stakeholders in the region and continued notwithstanding the difficulties posed by the pandemic. The submission to the government was made in April and an external review panel completed its evaluation in mid-June of AY '20-'21.

\section{Proportional Implementation of Quality Processes}

The quality framework also includes the academic activities of the institute, including course design and approval, the setting and monitoring of standards, the making of awards and the academic integrity of the learner and learning process. Increasingly, these regulations must also account for different types of learners, including new modes of delivery (such as technology enhanced learning, including on-line delivery), as well as the diversity of types and backgrounds of learners.

It was vital therefore that the quality framework be dynamic, flexible and responsive in the pandemic period. For example, the validation of new awards was carried out online in a manner that met the needs of quality assurance and the expectations of the award proposers. Virtual participation on a review panel, removing the requirement to travel to our institute, was seen as a positive by external panelists and may be retained in some instances post-COVID. While validation processes were not possible in the immediate aftermath of the first lockdown, they moved relatively quickly online, and the number of validation processes has been comparable to previous years.

\section{Comprehensive, Transparent and Publicly Accountable QA}

The institute is a full and committed member of the European Higher Education Area and Bologna process, and as such, benchmark their approach to quality assurance against the ESG and other comparable systems of international effective practice.

The validation and awarding procedures were still consistently comprehensive, transparent and publicly accountable, which for example meant that procedures and evaluations of new award proposals continued to be published online.

Online publication was arguably more important due to limited campus access, so the institute also published its own self-reflective report for the institutional review, as well as the expert panel report from that review. The self-evaluation report had not been published for previous institutional quality reviews.

The student survey results and analysis for AY '19-'20 and AY '20-'21 are presented in brief here in FIGURES 1 and 2. These are discussed internally in Academic Council and individual departments and also made available to the institute community and wider public. 


\section{Monitoring and Measurement}

Measurement, reporting and publication are key aspects of good academic governance. The institute continued throughout the pandemic to measure and monitor performance, for example in new student applications, exam attendance, monitoring the transition online through indicators such as virtual learning environment page-hits and internal reviews of new and modified programs.

Accountability, control and scrutiny were ensured through reporting, student surveys, program and School Board reports, new program and program change panel review reports, postgraduate vivas, meeting the national statutory quality body in a Dialogue Meeting, and maintaining transparency of all framework actions through publication on the institute website.

Emergency Remote Teaching required an immediate and sustained response in the institute's Virtual Learning Environment, Moodle. Since March in AY '19-'20, Moodle has been available throughout, with $100 \%$ Moodle Uptime. Unsurprisingly, Moodle Traffic increased, up to $157 \%$ in the March-May period compared with similar months in 2019. The daily average of just under 60,000 Moodle pageviews in the AY '19-'20 March-May period has since was immediately exceeded on return to education in September 2020 , where the resumption of classes saw the number of pageviews soar to new records of over 200,000.

Student feedback and participation is an important aspect of the capacity of the institute to assess its performance. The entire student body was surveyed in May of AY '19-'20 $(\mathrm{N}=1,300)$ and again in May AY '20-'21 (N=800), with the results shown in FIGURE 1 and compared with the combined results for the previous 3 years $(\mathrm{N}=2,500)$. Students were asked to rank each category from 1 to 4 on a Likert scale, with 1 being lowest and 4 highest. The results presented are the average scores calculated from all student responses.

\section{FIGURE 1 STUDENTS’ IMPRESSIONS OF THEIR PROGRAMME}

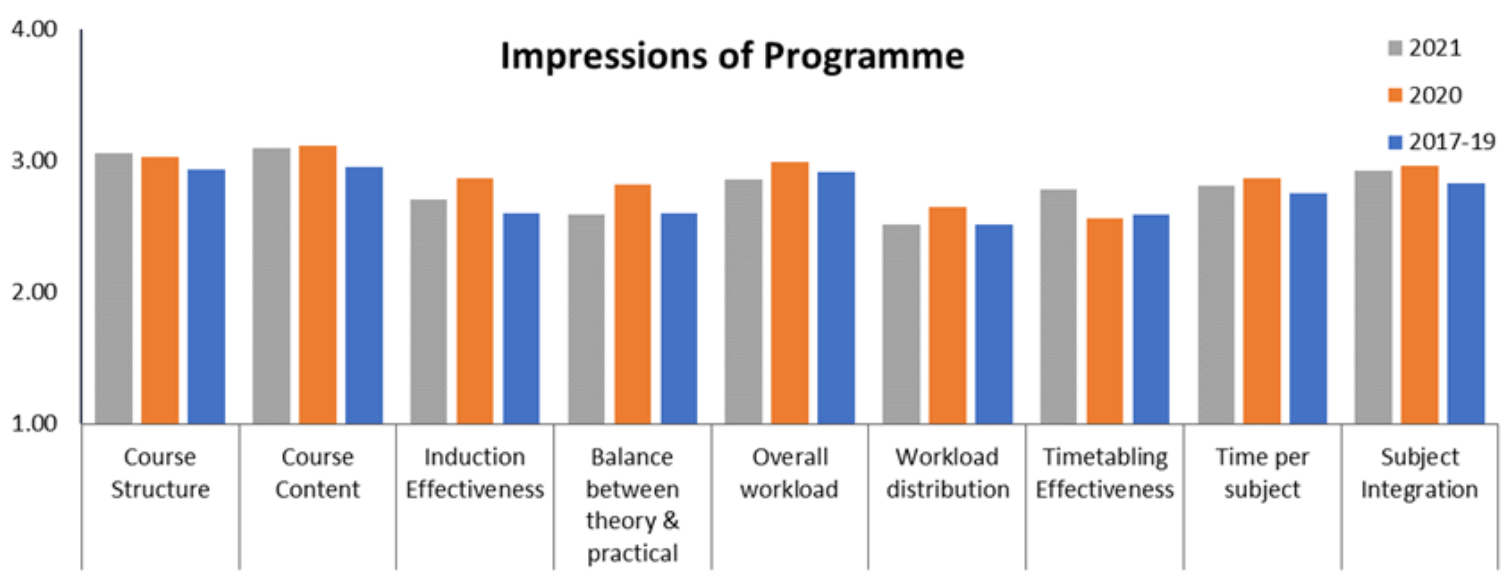

In the immediate aftermath following closure the AY '19-'20 students were broadly more positive in their program impressions than in previous years. However, the response in AY '20-'21, while still broadly positive, was more nuanced. They were less impressed with induction effectiveness, notwithstanding new approaches taken nor with the balance between theory and practical work, perhaps because access to practical work was severely restricted. One area of ongoing concern has been to find a new balance in students' workload, and this is an area that may require more consultation.

In spite of improved efforts to communicate with students, the AY '19-'20 students overall felt that this was an area that had to improve in AY '20-'21, more or less across every single category with the exception of Project Support and Continuous Assessment feedback, as can be seen in FIGURE 2. AY '20-'21 was predominantly online and presented more challenge and complexity than the previous year. Nonetheless 
the new approaches adopted have been scored highly in the AY '20-'21 survey, with high scores in every category, bar continuous assessment feedback, which fell back to the previous years' ranking.

\section{FIGURE 2 \\ EFFECTIVENESS OF COMMUNICATION}

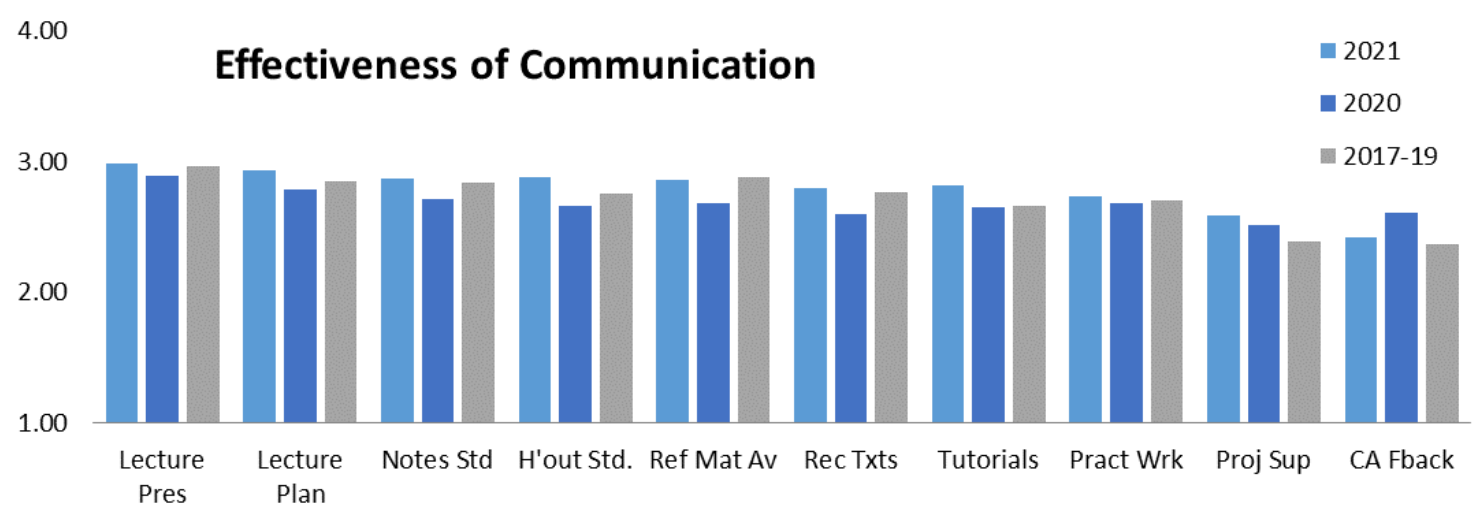

\section{CONCLUSION}

COVID-19 tested the quality framework of our higher education institute and in this paper an entire year of operation has been considered. Overall, the framework in place was sufficiently robust to deal with all academic, regulatory and financial demands placed on it, although the implementation of the framework had to be modified in several areas.

Evaluation of performance was provided by the authors, the students, external examiners and an external QA review panel. The institute student was involved in addressing the challenges from the beginning, through their students' union representatives, and also through a survey of the entire student body. Overall, the students were happy with the institute's response, but also offer indications where further improvement is required.

The Quality Assurance Framework was not designed for a pandemic scenario but operated effectively during COVID-19. While the move to social distancing was initially a challenge, quite soon operations were continued as before, including new policy development; academic measurement, reporting and publication and oversight of emergency remote learning and teaching.

\section{REFERENCES}

Burki, T.K. (2020, June). COVID-19: Consequences for higher education. The Lancet Oncology, 21(6), 758.

Camilleri, M. (2021). Evaluating service quality and performance of higher education institutions: A systematic review and a post-COVID-19 outlook. International Journal of Quality and Service Sciences, 13(2), 268-281.

Crawford, J., Butler-Henderson, K., Rudolph, J., Malkawi, B., Glowatz, M., Burton, R., .. . Lam, S. (2020). COVID-19: 20 countries' higher education intra-period digital pedagogy responses. Journal of Applied Learning \& Teaching, 3(1), 1-20.

Du, C., Zan, M., Cho, M., Fenton, J., Hsiao, P., Hsiao, R., . . Tucker, R. (2021). The Effects of Sleep Quality and Resilience on Perceived Stress, Dietary Behaviors, and Alcohol Misuse: A Mediation-Moderation Analysis of Higher Education Students from Asia, Europe, and North America during the COVID-19 Pandemic. Nutrients, 13, 442.

García-Peñalvo, F.J., Corell, A., Abella-García, V., \& Grande, M. (2020). Online assessment in higher education in the time of COVID-19. Education in the Knowledge Society, 21. 
Kumar Arora, A., \& Srinivasan, R. (2020). Impact of Pandemic COVID-19 on the Teaching - Learning Process: A Study of Higher Education Teachers. Prabandhan: Indian Journal of Management.

Kumar, R. (2020). Assessing Higher Education in the COVID-19 Era. Brock Education: A Journal of Educational Research and Practice, 29(2), 37-41.

Lancet. (2020). Research and higher education in the time of COVID-19. The Lancet, 396.

Marinoni, G., van't Land, H., \& Jense, T. (2020). The impact of COVID-19 on Higher Education. Paris: International Association of Universities.

Martel, M. (2020). COVID-19 Effects on U.S. Higher Education Campuses: Academic Student Mobility to and from China. The Power of International Education.

Mishra, L., Gupta, T., \& Shree, A. (2020). Online teaching-learning in higher education during lockdown period of COVID-19 pandemic. International Journal of Educational Research Open, 1.

Neuwirth, L.S., Jovic, S., \& Mukherji, B.R. (2020). Reimagining higher education during and postCOVID-19: Challenges and opportunities. Journal of Adult and Continuing Education.

O'Leary, P., \& O’Byrne, D. (2021, June). Evaluation of a Higher Education institute's Quality Framework performance during COVID-19. Higher Education Advances 2021 (HEAd'21). Valencia, Spain.

Ramírez-Hurtado, J., Hernández-Díaz, A., López-Sánchez, A., \& Pérez-León, V. (2021). Measuring Online Teaching Service Quality in Higher Education in the COVID-19 Environment. International Journal of Environmental Research and Public Health, 18(5).

Toquero, C.M. (2020). Challenges and Opportunities for Higher Education Amid the COVID-19 Pandemic. The Philippine ContextPedagogical Research, 5(4), Article em0063. 